\title{
Frequency conversion control method for reducing switching loss of inverter
}

\author{
Shixian Li1, a, Fanghua Zhang ${ }^{1, b}$ \\ ${ }^{1}$ Nanjing University of Aeronautics \& Astronautics, Nanjing, China \\ alsx7@sina.com, bzhangfh@nuaa.edu.cn
}

\begin{abstract}
Keywords: Inverter, frequency conversion, loss optimization, dual Buck converter.
Abstract. Inverter with sinusoidal pulse width modulation in the operation of certain moments of the inductor current ripple is smaller, still maintain a switch with higher frequency, which will undoubtedly increase the loss of inverter unnecessarily. In this paper, a new control method of the inverter switching frequency is proposed. In this method, inverter switching frequency is modified, while inductor current ripple peak to peak value is kept constant, in order to reduce the switching loss of the inverter power devices. Small signal modeling and analysis show that the introduction of inverter switching frequency control will not affect the steady-state and dynamic performance of the loop control system. The effectiveness of the proposed control method is verified by both simulation and experiment.
\end{abstract}

\section{Introduction}

The switching losses of the power devices will increase for MW inverter using IGBT or IGCT, with the increase of the application power and voltage. Switching frequency is generally less than $1 \mathrm{kHz}$, in order to satisfy the lifetime and switching losses of IGBT or IGCT ${ }^{[1-2]}$.

Reducing the switching frequency under hard switching conditions is obviously helpful to improve the efficiency, but it may bring about problems. If the design parameter of filter is not changed, the inductor current ripple will become large, instability and greater THD of the system will be caused bring. Domestic and foreign experts and scholars have carried out a lot of research on the driving technology of high power inverter, developing a lot of inverter control methods, effectively reducing the switching frequency. At present, the method which can effectively reduce the switching frequency without increasing the current distortion is mainly used in synchronous optimal pulse width modulation strategy. Although the control method is widely used, the calculation of the switching angle is complex, which is hard to be done online, and has limited dynamic response ${ }^{[3]}$.

In this paper, a novel frequency conversion control method is proposed to reduce the switching loss of inverter. THD and stability of inverter system are not influenced in this method. The effectiveness of the proposed control method is verified by both simulation and experiment.

\section{Reducing the switching frequency of the inverter can reduce the switching loss}

Dual Buck inverter can be used SPWM control method ${ }^{[4]}$. As is shown in Fig. 1, when the dual Buck inverter output voltage is close to the input voltage, the inductor current ripple is small. Therefore, as is shown in Fig. 2, switching frequency can be reduced at this stage appropriately, in order to reduce the switching losses and improve inverter efficiency. 


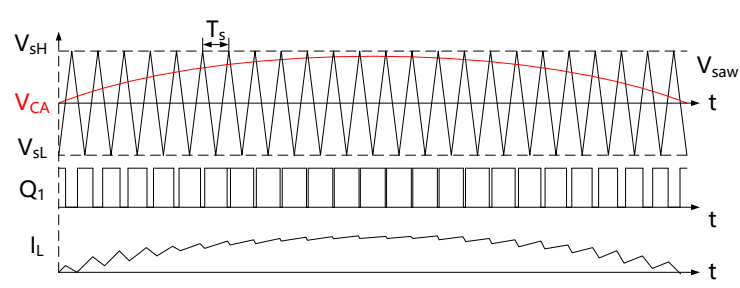

Fig. 1 Traditional dual buck inverter drive control waveform

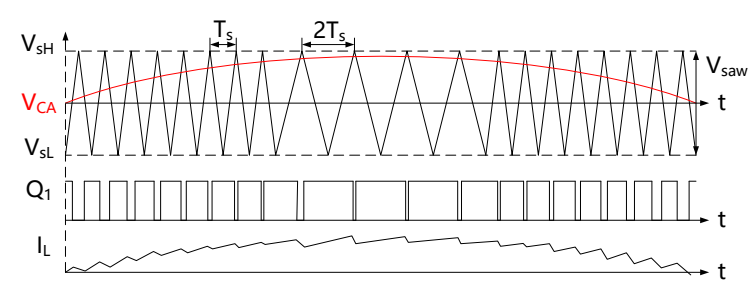

Fig. 2 Variable frequency control dual Buck inverter drive waveform

\section{Switching frequency control method for reducing switching loss of inverter}

This inverter control system is based on the DSP28335. whose switching frequency is $80 \mathrm{kHz}$, and output is $400 \mathrm{~Hz}$ sine wave. In this method, the switching cycle is always integer times of $12.5 \mathrm{uS}$ (corresponding to the $80 \mathrm{kHz}$ frequency), considering the calculation accuracy of the digital chip and difficulty of frequent regulation of PI.

The peak to peak value of the inductor current ripple is calculated:

$V_{p p}=D *(1-D) U_{i n} * T / L$

When $\mathrm{D}=0.5$, $V_{\text {ppmax }}=0.25 * \operatorname{Uin} * T / L$

It can be deduced that $V_{p p}<V_{p p \max } / 2$ when $D>(2+\sqrt{2}) / 4$ or $D<(2-\sqrt{2}) / 4$,

Because switching losses are proportional to the product of Uin and iL. When the output voltage is $U_{o}>(2+\sqrt{2}) V_{\text {in }} / 4$ or $U_{o}<(2-\sqrt{2}) V_{i n} / 4$, the switching frequency is halved, so the switching loss is halved. Product of Uin, iL and the weight value is accumulated with in a cycle. According to the load impedance angle, Uin and iL have distinct phase difference. Optimization curve of efficiency relating to the load impedance angle is shown in Fig. 3.

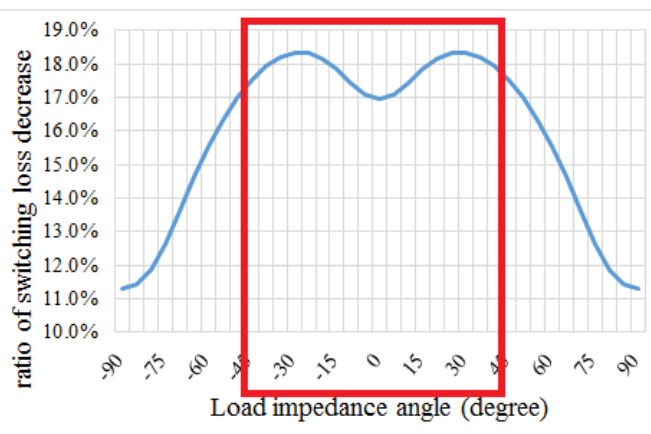

Fig. 3 Load impedance angle and switching loss reduction ratio

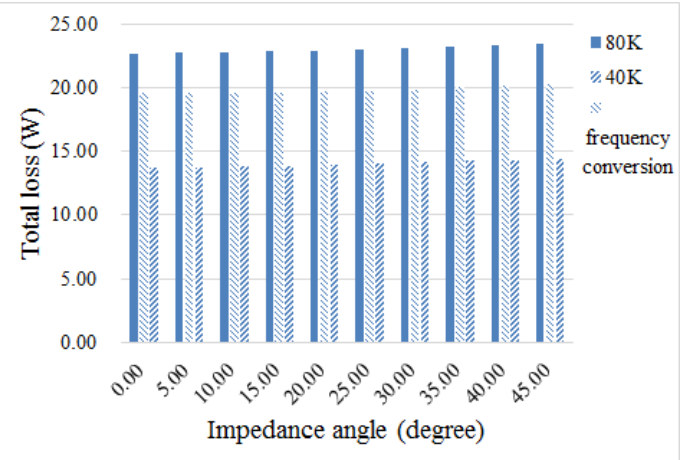

Fig. 4 Inverter loss ratio under different load impedance angle

It can be seen from Fig. 3 that in the range of 45 to 45 DEG of load impedance angle the switching frequency can be reduced when inductor current ripple is small, so that switching losses can be reduced from $16.94 \%$ to $18.32 \%$ compared to the conventional control method. Moreover, because the maximum peak to peak value of inductance current ripple is not changed, the requirements of the filter are completely unchanged.

There is no necessary to reduce the switching frequency to lower than $40 \mathrm{kHz}$, when inductor current ripple is smaller. Otherwise, the lower switching frequency will cause the system unstable, but cannot raise efficiency, and will also make the digital control method more complex.

\section{Loss analysis}

A new method of calculating the inverter loss is proposed in the paper ${ }^{[5]}$, which can calculate the inverter loss more accurately. 
Under different load conditions, the switching frequency is $80 \mathrm{kHz}, 40 \mathrm{kHz}$ and frequency conversion control of $80 \mathrm{kHz}$, The calculation results of losses are shown in Fig. 4 and Fig. 5:

Specific optimization contrast and data as shown in Fig. 5. It shows that under different load conditions, the frequency conversion control method reduces the switching losses of inverter by $15.95 \%-18.33 \%$, compared to the $80 \mathrm{kHz}$ switching frequency using the method of conventional control method. In the case of light load inverter, optimal proportion of switching losses decreases slightly.

Efficiency curve of the whole machine under different load is shown in Fig. 6.

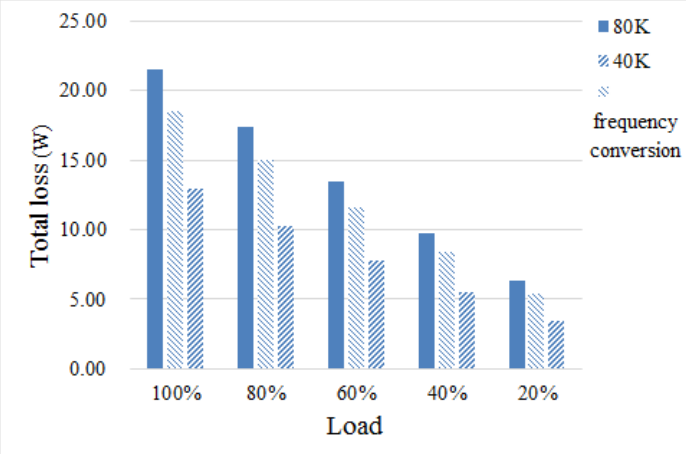

Fig. 5 Inverter loss under different load

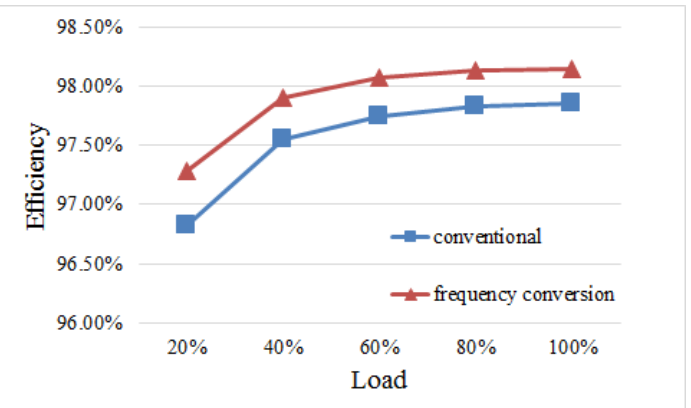

Fig. 6 Efficiency curve under different load

\section{System control bandwidth analysis}

The system diagram of frequency conversion control is introduced in Fig. 7. Transfer function of control system is shown in equation (1), where $G_{z}(s)$ is the transfer function for zero order holder; $G_{f}(s)$ is the transfer function of duty ratio to output voltage and $G_{s}(s)$ is transfer function for voltage regulator; $H_{g}(s)$ is sampling coefficient of output voltage, $K$ is frequency modulation FM coefficient, and Vref is the reference signal of output voltage. According to the control flow graph, the inverter switching frequency conversion control of the loop closed loop gain $\mathrm{Gb}(\mathrm{s})$ is (1):

$$
G_{b}(\mathrm{~s})=\frac{G z(s) G s(s) G f(s)}{1+G z(s) G s(s) G f(s) H g(s)}
$$

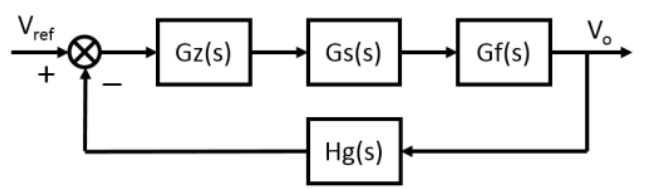

Fig. 7 Dual buck inverter control block diagram The double Buck converter is set under CCM mode, Gz (s), Gs (s), Gf (s) and the ratio of $\mathrm{K}$ and $\mathrm{Hg}$ (s) link design are calculated in the formula, set the modulation frequency modulation coefficient $\mathrm{K}$ to introduce respectively $\mathrm{K}=1$ and $\mathrm{K}=2$, get into the system before and after the loop gain output voltage feed forward by Mathcad software, as shown in Fig. 8 and Fig. 9. We can see from Fig. 9, introduced the inverter switching frequency control and coincident loop gain curve of the system, namely the inverter switching frequency control is introduced will not affect the steady-state and dynamic performance of the original double Buck inverter control system.

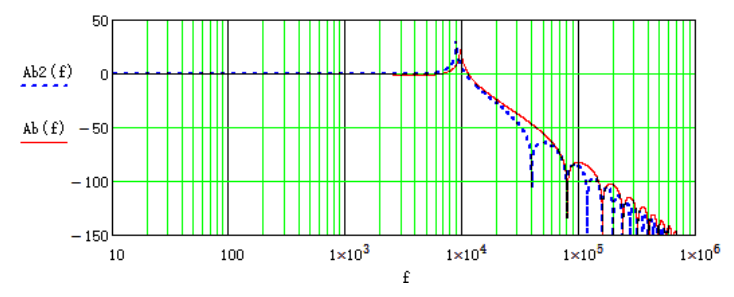

Fig. 8 Comparison of closed loop gain at different sampling frequencies

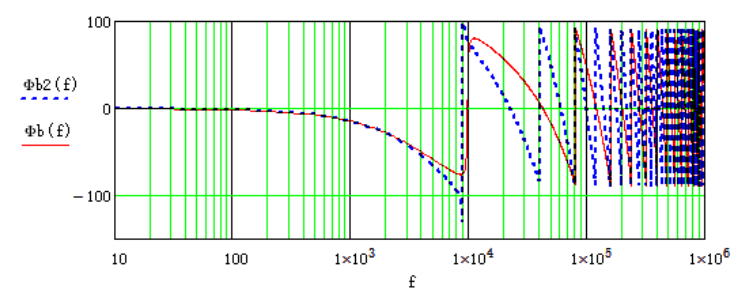

Fig. 9 Phase-angle of the closed-loop-gain at different sampling frequencies 


\section{THD impact analysis}

The effect of switching frequency on the inverter output voltage waveform THD is reflected in the change of the switching frequency. When the switching frequency is $80 \mathrm{kHz}$, the square wave before the LC filter mainly contains the $80 \mathrm{kHz}$ harmonics, whose RMS value is $U_{\text {in }}$. As the inverter switching frequency is $40 \mathrm{kHz}$ which mainly contains $40 \mathrm{kHz}$ harmonics. In Fig. 8, the Bode diagram can be seen when the sampling frequency is $80 \mathrm{kHz}$ and $40 \mathrm{kHz}$. The corresponding loop gain is $-75 \mathrm{~dB}$ and $-55 \mathrm{~dB}$ respectively. According to the definition of the single harmonic content SHD:

$$
S H D_{n}=Q_{n} / Q_{1}
$$

Get $S H D_{80 k}=0.028 \%$ and $S H D_{40 k}=0.28 \%$. Switching frequency of the output voltage of the change will bring the maximum increase of $0.25 \%$, the value of this increase in the inverter system is very small, and you can ignore the increase of THD.

\section{Simulation and experimental verification}

The main parameters of the dual Buck inverter prototype are the same as the PSIM simulation parameters. The MCU chip is TMS320F28335.

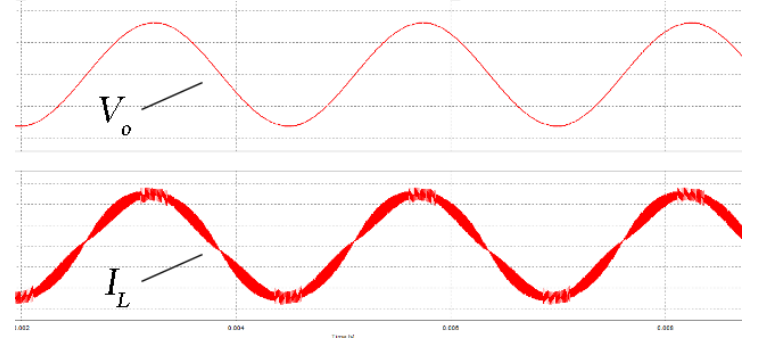

Fig. 10 Frequency conversion control inverter simulation waveform

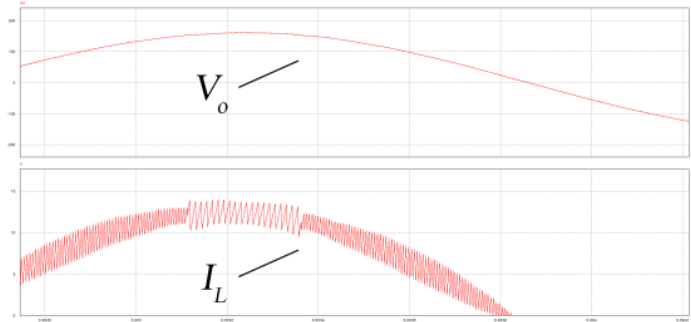

Fig. 11 Inductor current and voltage simulation waveforms

Fig. 12 and Fig. 10 shows the inductor current and output voltage waveforms with conventional inverter control method and frequency conversion control method. Fig. 11 is a partial view of the voltage and current waveforms in Fig. 10.

Fig. 13 and Fig. 14 show the experimental results of the inductor current and voltage waveforms of the inverter respectively. The drive voltage $u_{G S}$ of $\mathrm{Q}_{1}$ and $\mathrm{Q}_{4}$ is shown in Fig. 15. And through the oscilloscope to save the waveform file DFT analysis of the output voltage of the THD. Fig. 13 and Fig. 10 shows that the experimental results and simulation analysis results, the introduction of inverter frequency conversion control method does not affect the stable operation of the inverter the maximum ripple peak inductor current. Furthermore, this novel control method has little impact on the waveform quality THD, but can effectively reduce the switching losses of the switch. 


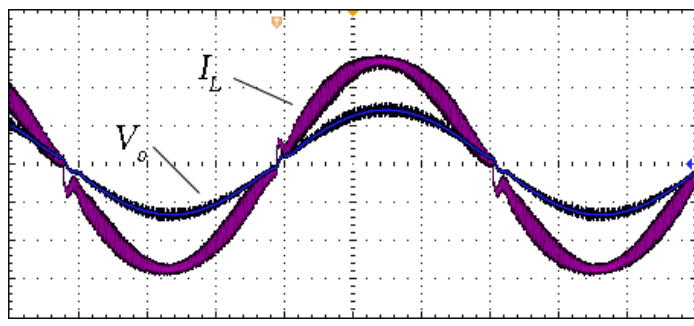

Fig. 12 Experimental waveforms of conventional inverter control methods

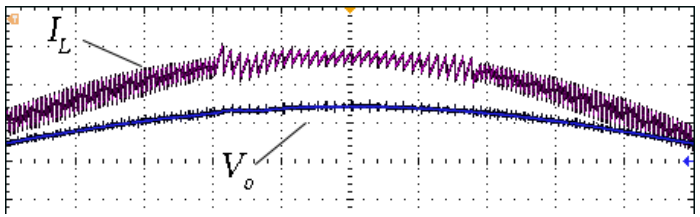

Fig. 14 Inductor current and voltage experimental waveform

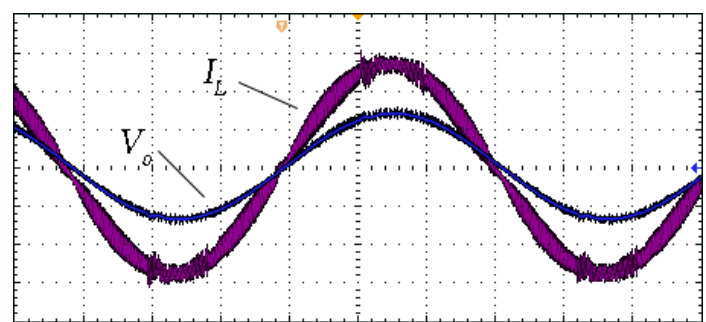

Fig. 13 Switching frequency control method of inverter experimental waveform

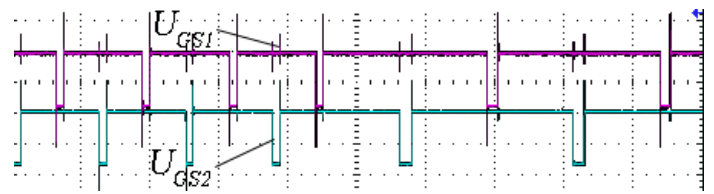

Fig. 15 Drive waveforms of switching tube Q1 and Q4

Experimental tests is carried out under different load conditions. THD of the waveform is calculated through the oscilloscope picture. The calculation results are shown in Fig. 16 and Fig. 17, and although the simulation results have certain discrepancy, which is due to the The experimental conditions are not as good as the simulation conditions, but you can see the trend of change is consistent, and the gap of THD between the traditional control method and frequency conversion control method are relatively consistent with the simulation results.

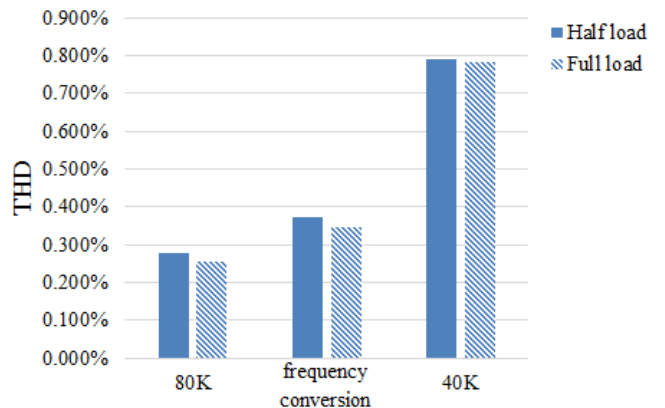

Fig. 16 THD under different control methods (simulation)

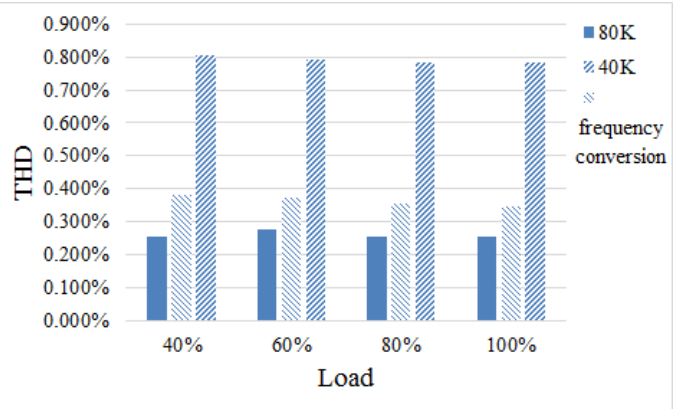

Fig. 18 THD under different control methods

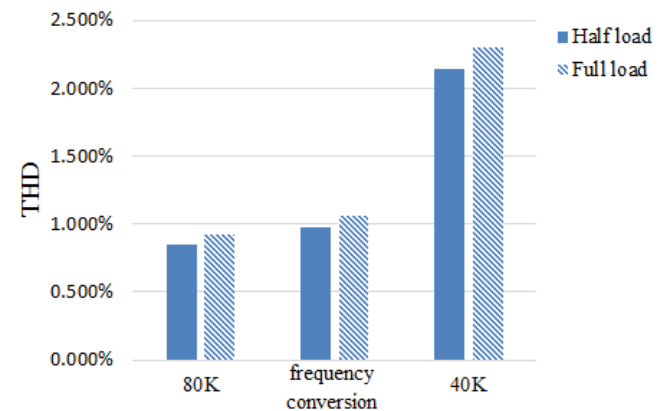

Fig. 17 THD under different control methods (experimental)

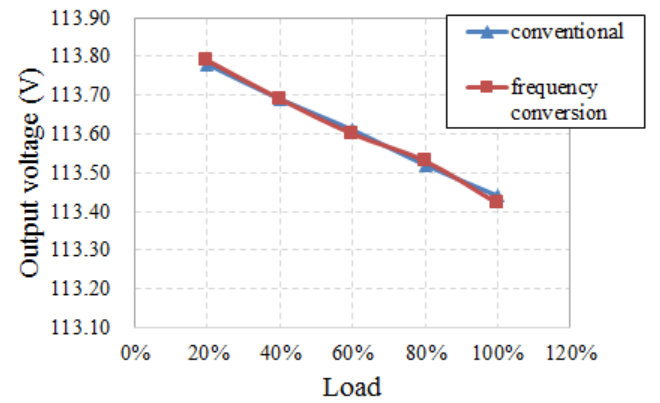

Fig. 19 Dual Buck inverter load regulation rate

Fig. 18 shows simulation values of THD under different loads using $80 \mathrm{kHz}$ switching frequency control, frequency conversion control and $40 \mathrm{kHz}$ switching frequency control of the inverter. As you can see, although the switch inverter waveform quality, in this proposed control method, decreases slightly compared with the conventional control method, but still more than the situation that switching frequency is reduced in the whole cycle, and the switching losses of the device is reduced by $15.95 \%-18.33 \%$. 


\section{Steady state performance verification}

Fig. 19 shows the load regulation rate of double Buck inverter before and after the output voltage feed forward. It can be seen that the output voltage feed forward will not affect the steady-state performance of the original control system. (The output voltage is calculated by the waveform of the oscilloscope waveform is effective to calculate the fundamental wave)

\section{Conclusions}

This paper analyzes the common control methods of reducing the inverter frequency, a frequency conversion control method applied in the operation process of the inverter is proposed in this paper. This paper gives reasonable coefficient of frequency conversion and parameter design method of the system that does not significantly affect the quality of the output voltage waveform. After theoretical analysis and simulation experiments, the following conclusions can be drawn:

(1) The frequency conversion control method proposed in this paper can effectively reduce the switching losses of power devices.

(2) Frequency conversion control method does not affect the steady-state and dynamic performance of the original closed loop system.

\section{References}

[1] Geyer T.A comparison of control and modulation schemes for medium voltage drives: Emerging predictive control concepts versus PWM-based schemes[J]. IEEE Transactions on Industry Applications, 2011, 47(3): 1380-1389.

[2] Mertens A, Sommer R, Brunotte C.Applications of medium voltage drives with IGBT three-level inverter[C]. IEE Seminar on PWM Medium Voltage Drives . Birmingham, UK: IEE, 2000: $7 / 1-7 / 4$.

[3] QI Xin, ZHOU Ke, WANG Changsong, ZHOU Xiaomin, PAN Zhiyun, MA Xianghua. Control Strategies for Medium and High Power AC Machine Inverters at Low Switching Frequencies An Overview [J]. Proceedings of the CSEE,2015,24:6445-6458.

[4] Su T, Zhang F, Xie J, et al. A high power density dual-buck full-bridge inverter based on carrier phase-shifted SPWM control[C]. Applied Power Electronics Conference and Exposition (APEC), 2015: 1715-1721.

[5] HONG Feng, SHAN Ren-zhong, WANG Hui-zhen, YAN Yang-guang. Analysis and Calculation of Inverter Power Loss [J]. Proceedings of the CSEE,2008,15:72-78. 